\title{
NEW APPROACHES TO DIAGNOSTICS OF C.DIFFICILE INFECTION
}

\author{
Stofkova $Z^{1}$, Novakova $E^{2}$, SADLOŇOVÁ, $V^{3}$ \\ ${ }^{1}$ Department of Microbiology and Imunology, Jessenius Faculty of Medicine in Martin, \\ Comenius University in Bratislava, external PhD student \\ ${ }^{2,3}$ Department of Microbiology and Imunology, Jessenius Faculty of Medicine in Martin, \\ Comenius University in Bratislava
}

\begin{abstract}
A b s tract
Introduction: Clostridium difficile infection (CDI) is the most common infectious cause of diarrhoea in hospitalised patients. It is a severe issue with increasing incidence in healtcare facilities and commmunities. Early and appropriate testing of CDI is very imortant. There are many different approaches that can be used in the CDI laboratory diagnostics. Recently performed studies evaluated diagnostic algorithms to optimize laboratory CDI diagnostics. The aim of the work was to implement an optimal algorithm of testing methods in CDI diagnostics.

Materials and methods: The retrospective analysis of patients' samples suspected on CDI was carried out in 2017. The data was analysed from the Laboratory Klinicka Biochemia in Zilina. The application of ESCMID recommendation criteria for algorithm of testing, together with procedures in laboratory practice was carried out.

Results: The analysis of the data showed that the applied algorithm or testing in CDI and highlighted the importance of preanalytic phase, and the evaluation of testing methods and results, together with clinical findings. Two- and three- step algorithm together with cultivation methods were applied. 87,6\% of samples could be evaluated with positive or negative results according to the 2-step algorhitm. Further samples were assessed and tested by ELISA test or PCR method.

Conclusion: CDI represents a significant burden in healthcare facilities. An early diagnostics and interpretation helps to start the early treatment and helps in surveillence of the infection.
\end{abstract}

Key words: C.difficile infection, laboratory diagnostics, testing algorithm

\section{INTRODUCTION}

Clostridium difficile infection (CDI) is the most common infectious cause of diarrhoea acquired in health care facilities. Approximately 15-25\% of all cases of antibiotic-associated colitis are caused by $C$. difficile and this probability increases with the severity of disease, reaching 95-100\% among patients with documented antibiotic-associated pseudomembraneous colitis (PMC). [1]

Recent studies have shown increasing incidence of CDI. For example, the incidence of healthcare acquired CDI increased 2 to 2.5 times from the late 1990s to the early 2000s, and even more in elderly people. [2] The CDI is responsible for significant morbidity of patients and incidence continues to rise. From 2010 to 2017 increased the incidence in Slovakia from 0.9 to $20.6 / 10.000$ of hospitalised patients. In study carried out by Novak et al. $85 \%$ were $\mathrm{HAI}-\mathrm{CDI}$ acquired and 15\% were CA-CDI acquired. [3]

The management of CDI is complex and comprises clinical, laboratory and radiological diagnosis. CDI still remains a significant healthcare burden in health care institutions. Accurate and fast diagnostics of CDI is essential for optimal patient care and prevention of the spreading of the infection. [4]

Corresponding author: Assoc. Prof. MUDr. Elena Novakova, PhD; e-mail: elena.novakova@jfmed.uniba.sk (c) 2020 Elena Novakova et al.

This work is licensed under the Creative Commons Attribution-NonCommercial-NoDerivs 4.0 License (https://creativecommons.org/licenses/by-nc-nd/4.0/) 
C. difficile is a minor part of the natural intestinal flora in the colon. By applying antibiotics during treatment, it becomes the predominant type of intestinal flora. Primary factors of $C$. difficile virulence are 2 toxins, toxin A (TcdA) and toxin B (TcdB). [5]

Toxin A is an enterotoxin and toxin B is a cytotoxin induces depolymerization of actin. [6] New hypervirulent strain (i.e. 027/NAP1/BI ) produce besides 2 large clostridial toxins also binary toxin. In several studies carried out recently ribotype 027, 176 and 001 were determined predominantly in Slovakia. [7] [8]

The interplay between the pathogenic virulence factors of the bacterium and the immune response of the host may in part explain how colonisation with C.difficile can result in wide spectrum of outcomes. [9] [10] Pseudomembranous enterocolitis may result in toxic megacolon, ileum, or rupture of the intestine, endangering the patient's life. [11]

Colonization with $C$. difficile does not automatically lead to development of symptomatic CDI. Colonization rates in healthy humans in the community range from $0.8 \%$ to $13 \%$ and are higher in long-term care facility residents [6]. However, there are strains of C. difficile, which do not produce any of the toxins and are unable to induce the disease. [11] From these C.difficile strains 20-25\% may be non-toxigenic. [12]

Recently, studies have been carried out in order to evaluate diagnostic algorithms to optimize CDI laboratory diagnostics. Due to the different sensitivity of the different methods used, a combination of at least 2 different tests is preferred according to current recommendations, with the determination of toxins A and B considered to be an optimal test. [13]

In CDI diagnosis, the use of two- or three-step algorithms are recommended by ESCMID (European Society for Clinical Microbiology and Infectious Diseases) in which a positive test is confirmed by one or two confirmatory tests or by a reference method. The algorithm can start with GDH-EIA (glutamate dehydrogenase - enzyme immunoassay) or NAATs (nucleid acid amplification test) - e.g. real-time PCR. The positive results should be confirmed by toxin A/B enzyme immunoassay (EIA). Samples confirmed by this second toxin A/B EIA test can be confirmed as CDI positive, Fig. 1 .

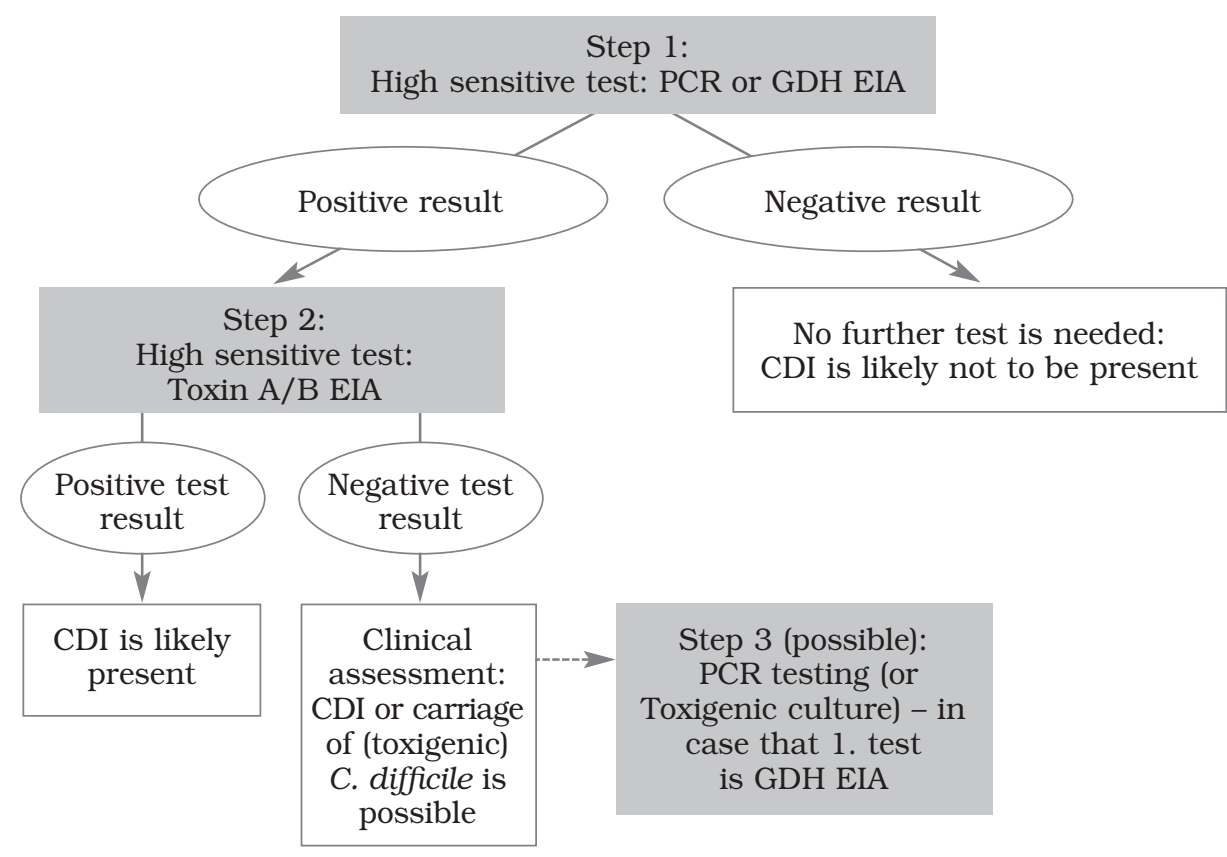

Fig. 1 Algorithm of diagnostics of CDI

Source: Crobach et al. [13] 
Samples evaluated as negative with GDH-EIA or PCR, can be assessed as CDI negative.

If the GDH test was performed as the first test, it is possible to carry out PCR test as a third step. In the case of discrepant testing results, a confirmatory test can be performed, e.g. by the real-time PCR method. PCR-based testing is unable to differentiate infection and colonisation. As a result, reliance on it as a stand-alone test may result in over-diagnosis. The low sensitivity of toxin EIAs also makes these tests unsuitable as stand-alone tests. [13] [14]

\section{MATERIALS AND METHODS}

The retrospective analysis of patients` biologic samples suspected on CDI was carried out during 2017. The data were analysed by the Laboratory Klinicka Biochemia, Zilina.

The implementation of testing algorithm according to ESCMID recommendation criteria, together with procedures in laboratory practice were presented. The comparison of several testing methods was carried out. All biologic specimens received in the laboratory for diagnosis of CDI were tested in in- and outpatients.

Stool samples suspected on $C$. difficile infection were tested by direct testing methods for detection of genes, enzymes, toxins in stool. Testing method in the diagnostics of CDI included: detection of exoensyme Glutamate Dehydrogenase (GDH) by (EIA BioTecCER TEST), detection of $C$. difficile toxins A/B by immunoenzymatic assays (EIA BioTecCER TEST), immunoenzymatic methods for the detection of toxins A/B by Enzyme Linked Immunosorbent Assay (ELISA - ProSpecT C.difficile Toxin A/B Microplate Assay) and nucleic acid amplification test - real-time PCR (for detection of B toxin gene(tcdB), binary toxin genes cdtA and cdtB and deletion at nucleotide 117) - (GenExpert Cepheid), anaerobic culture for $C$. difficile and identification of C.difficile strains by Matrix Assissted Light Desorption and Ionisation Time of Flight Mass Spectrometry (MALDI TOF MS) were applied.

\section{RESULTS}

During 2017-1923 specimen were delivered to the laboratory for C.difficile testing, 35 samples were excluded because of not meeting criteria of the sample size $(2 \mathrm{ml})$ or there were more samples delivered at the same time. The percentage of stool specimen from hospitalised patients included $93 \%$ and $7 \%$ of specimen was tested from ambulant patients.

The number of stool specimens were 1888 (1353 patients) were tested by at least two immunoenzymatic methods (immunochromatography for GDH and toxins A/B). 1144 stool stool samples were evaluated as negative $(60,6 \%)$.

Toxigenic strains of $C$. difficile were detected in 511 stool specimen (27\% of all specimen) and from 401 patients. In the studied sample positive specimens were in $54 \%$ from females (277) and $46 \%$ from males (234). An important risk factor for CDI was advanced age.

The 78,5\% (398) samples of patients were evaluated as positive in laboratory testing in the age group of patients with more than 65 years. The mean of tested samples was 1.5 samples per patient. Positive samples with GDH-EIA test and test for toxin A/B-EIA (two-step algorithm), according to the age group of the patients $(n=511)$ are displayed in Fig. 2 .

We applied the two- or three step algorithmic approach to C. difficile testing in order to reduce false positive and false negative results: two-step algorithm with detection of GDH-EIA (rapid test) and toxin A/B EIA (rapid test) - the combination of GDH with high sensitivity and rapid enzyme immunoassay test with relatively high specificity. In three-step approach were added the ELISA test for toxins A/B and the real-time PCR method in discordant cases (i.e. GDH positive samples and toxin negative samples in patients suspected of CDI). 


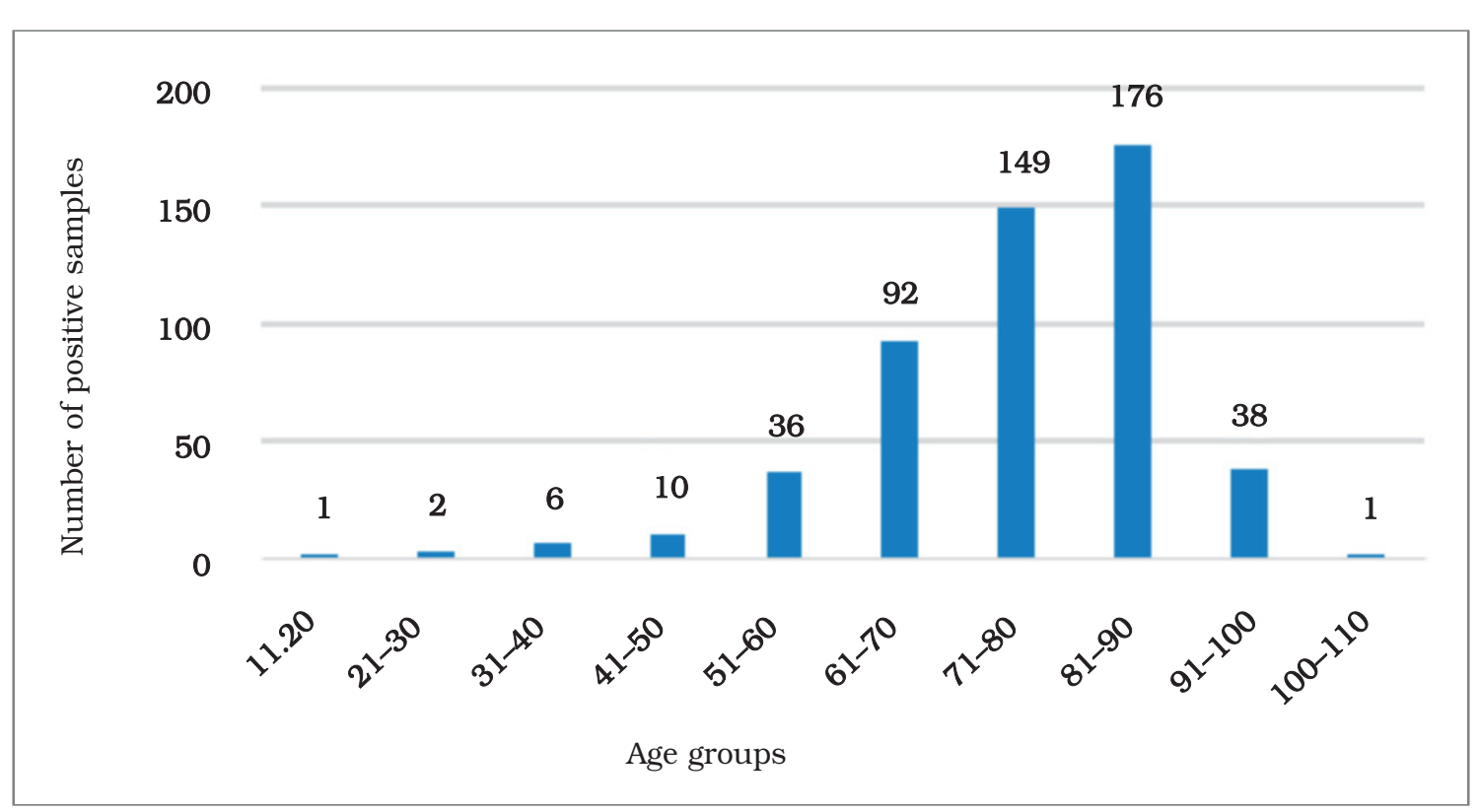

Fig. 2 Percentage of the positive samples by the 2-step algorithm

In this approach, the biologic samples were screened for the exoenzyme Glutamate dehydrogenase $(\mathrm{GDH})$ enzyme immunoassay and simultanously for rapid enzyme immunoassay for toxins A/B.

From the 1888 samples, 1144 samples (60 \%) were evaluated by the glutamate dehydrogenase test and rapid enzyme immunoassay for toxin A/B as negative. With negative results were tested 887 samples with GDH-EIA (rapid test), Toxins A/B-EIA (rapid test) and ELISA for toxins A/B. 257 biologic samples were tested negative with GDH-EIA and with Toxin A/B-EIA. The samples confirmed by these two rapid tests can be interpreted as negative without further examination (Fig. 3).

The patient stool samples were evaluated positively by Glutamate dehydrogenase (GDH-EIA and toxins A/B by enzyme imunoassay (EIA) by the two-step algorithm in 511 samples (401 patients). At the same time anaerobic cultivation have been performed subsequently to test the samples. The samples which resulted as positive in both rapid tests were then confirmed by enzymatic immunoassay (ELISA) - assay with higher specificity, samples were confirmed by these three methods in 504 samples.

Based on the two-step algorithm $87,6 \%$ samples could be evaluated as negative or positive and the results were sent on the day of testing (within 4 hours).

Samples with a GDH-positive result but with negative result for toxins A/B by rapid test (233) were tested by real-time PCR method. (Fig. 4). 31 samples were confirmed with PCR for the presence on B toxin gene (tcdB) or also on binary toxin genes (cdtA and cdtB) and deletion of tcdC gene in the nucleotide 117 . The laboratory results of PCR couldn't differentiate between CDI and colonisation by toxigenic C.difficile, but could confirm the presence of C.difficile toxigenic strain and exclude the presence of toxigenic strains in 65 samples, which were evaluated with negative results.

The immunochromatography assay for toxin A/B serves as a screening method and toxin could be presented under the detection limit in the biologic sample. From 136 samples, 26 samples were confirmed by ELISA test.

Samples evaluated positively for GDH and toxins A/B were tested for anaerobic culture for antimicrobial susceptibility. 


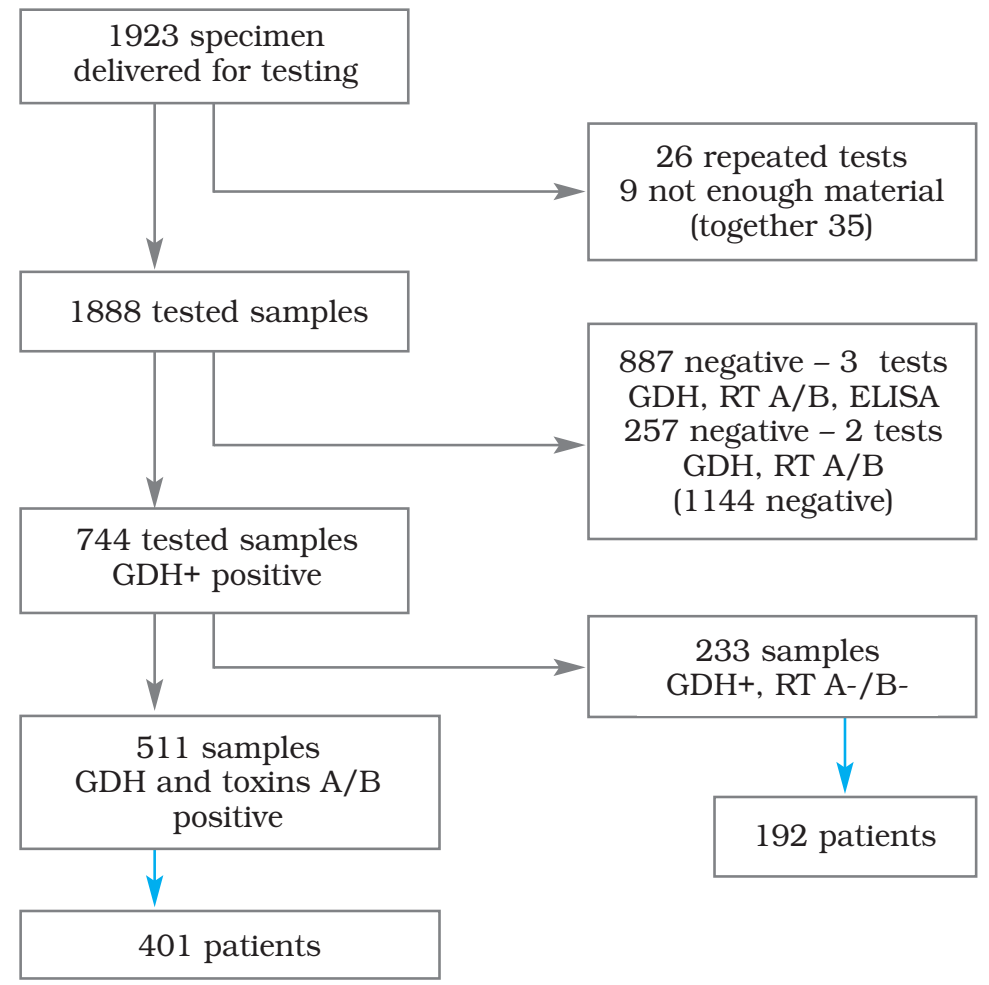

Fig. 3 Samples tested in laboratory in 2017

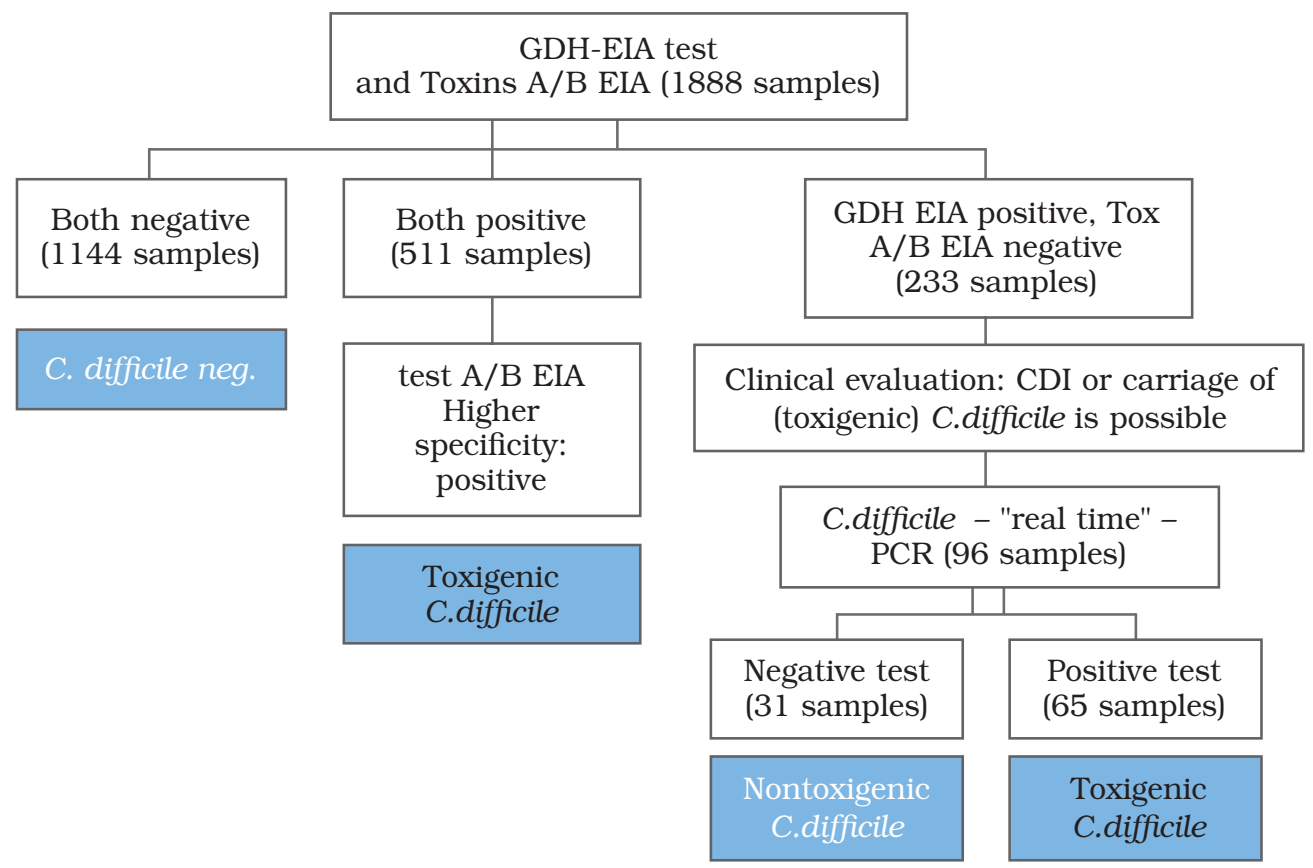

Fig. 4 Testing protocol (algorithm approach) applied in the laboratory practice for GDH-EIA and toxins A/B - EIA according to [13]) 


\section{DISCUSSION}

Differences in sensitivity and specificity, duration and cost of different test methods have resulted in laboratories using different testing algorithms. The optimal diagnostic approach to CDI diagnostics is still under discussion. A recent large study by Crobach et al. tried to establish the optimum diagnostic algorithm for CDI. [13]

Accurate diagnosis of Clostridium difficile infection (CDI) is crucial for proper treatment and for preventing the spread of the pathogen in healthcare facilities. The selection of diagnostic tests to confirm CDI is controversial because of the variety of laboratory methods that are available and used across various facilities, and lack of standard recommendations for testing. [15]

Due to the different sensitivity of the different methods used, a combination of at least 2 different tests is preferred according to current recommendations, with the determination of toxins A and B considered to be an optimal test. The advantage of an algorithm is that tests can be combined in such a way that the percentage of false-negative results can be decreased. [13]

A need for uniformity and standardisation in testing methods in CDI has been implicated in guidiance documents published by ESCMID and confirmed by various of studies all over the world. Variations in testing for C.difficile infection can hinder patient's care and increase the risk of transmission. [16]

An optimized diagnosis can be achieved by implementing a two- or three-step algorithm. Algorithms currently recommended by the ESCMID comprise a screening test with high sensitivity followed by a more specific test to detect free toxins. [4]

According to the Gilligan study, the two-step algorithm increased the capture rate of toxigenic strains by $40 \%$ compared to the separate detection of toxins by immunoenzymatic methods. [17] This fact was confirmed also by our findings in comparison with previous years. It could be also a result of implementation of algorithm in laboratory diagnosis but also due to better awareness of this issue, but also because of increasing incidence of the disease.

Within a two-step algorithm - samples tested by glutamate dehydrogenase (GDH) assay followed by toxins A/B by enzyme immunoassay (EIA), 80-90\% of the results may be evaluated with negative or positive result for up to approximately 4 hours. [18]

In our studied sample $87,6 \%$ samples could be tested with positive or negative results.

Therefore in the applied algorithm as a screening test there was applied detection of glutamate dehydrogenase (GDH) - the sensitivity is high, but specificity is lower because it is produced also in other Clostridia or nontoxigenic strains of C.difficile). The first test or GDH-EIA (PCR) has a high negative predictive value NPV, (negative predictive value) that reliably excludes non CDI patients. Therefore further test is needed for detection of toxins A/B by enzyme immunoassay (EIA), which has higher specificity according to the type of test. The limit of detection for the enzyme immunoassay test is the concentration of toxins in the biologic sample depending on the type of the test.

EIA for the detection of toxins A/B are considered less sensitive (50-80\%) for the detection of toxigenic C.difficile than the PCR method. Specificity of enzyme immunoassay (EIA) for toxins A/B is about 90-100\%, positive predictive value is high (99\%). Immunochromatography test for toxins A/B (membrane-type EIA) showed in our study sample slightly lower sensitivity as ELISA test (well-type EIA).

All laboratory tests have to be interpreted in relation to the clinical status of the patient and the risk factors for CDI. [11]

In our algorithm we applied GDH (membrane-type EIA) and toxins A/B - EIA (membranetype EIA) and ELISA test (well-type EIA) in order to exclude false positive and false negative results. The samples that were tested negative for both $\mathrm{GDH}$ and toxins A/B were classified as non-CDI. The samples that were evaluated as positive for GDH (membrane-type EIA) and toxins A/B (membrane and well-type EIA) were classified as CDI likely to be present. 
Interpretation of test results - if GDH (immunoenzymatic assay) or PCR positive and toxin ELISA are positive (PPV $=91.4 \%$ ) then $C$. difficile is most likely present. The result should be reported. If glutamate dehydrogenase GDH, (immunoenzymatic assay), or PCR positive and toxins A/B ELISA are negative, then $C$. difficile may be present, i. e. it is potentially C. difficile carrier - the results are not included in the mandatory reporting. If GDH (immunoenzymatic assay) or PCR method is negative and toxins A/B ELISA is negative (NPV = 98.9 \%) then it is very unlikely that $C$. difficile is present. [18]

The samples glutamate dehydrogenase $(\mathrm{GDH})$ - negative and toxins A/B - positive are rarely reported and they need to be retested. In the study, carried out by Krutova et al., after anaerobic cultivation samples were retested and were $\mathrm{GDH}$ - positive and toxins A/B - positive. [19] In our study samples glutamate dehydrogenase (GDH) - negative and toxins A/B (EIA) - positive were not detected.

For positive GDH tests and negative A / B toxin test assays, PCR testing is recommended to determine the presence of a toxigenic strain or toxigenic culture. [13]

$12,4 \%$ (233) samples were evaluated positive in glutamate dehydrogenase (GDH) test and on toxin A/B negative. In discordant cases, in suspition of CDI and positive symptoms, real-time PCR testing was applied. According to three-step algorithm - „real-time“ PCR - 31 samples (32.3\%) toxigenic or hypervirulent strains of C.difficile were excluded and 65 samples (67.7\%) C.difficile toxigenic or hypervirulent C.difficile strains were confirmed. The fast method enabled to set up the early onset of the treatment of CDI.

Several algorithms do not recommend „real-time“ PCR screening as a screening method for low-risk CDI patients because it is a highly sensitive method and can also identify patients who are colonized with C.difficile. However, it makes it very probable to exclude the presence of C.difficile, to determine and to distinguish the presence of toxigenic from a nontoxigenic strain of C.difficile.

The sensitivity of the test is defined as the probability that the test result will be positive for the person with the disease. The specificity of the test is defined as the probability that the test result is negative in a person without disease. [13] [20]

Preanalytic phase has to be also considered and can influence the final result of testing methods, since toxins are relatively unstable. Stool sample should be examined within 2 hours, or kept at about $5{ }^{\circ} \mathrm{C}$ for two days, otherwise it should be kept refrigerated at $-70^{\circ} \mathrm{C}$. [21]

Another issue is the number of samples per patient that should be submitted for testing. In our studied sample there was average testing rate lied on 1,5 per patient.

The diagnostic gain of repeat testing within a 7- day period with both toxins A/B EIA and PCR was demonstrated to be very low. However, in cases of ongoing clinical suspicion during an endemic situation, the submission of a repeat sample may be justified. [13]

The testing algorithm represents potential cost savings for laboratories. There are a number of factors that need to be considered, i.e. the prevalence of glutamate dehydrogenase (GDH) positive samples in a particular population. It should be noted that GDH assays are somewhat less sensitive than PCR methods. Each laboratory must perform an evaluation and assess a multi-step algorithm for its patient population. [22]

\section{CONCLUSION}

The best standard laboratory test for diagnostics has not been established, although many different approaches used in the laboratory diagnostics of CDI.

These results confirmed the importance of the pre-analytical phase and the need to evaluate laboratory results in relation to other laboratory findings regarding the sensitivity and specificity of tests used. The results confirmed that fast-track testing is a screening test for toxin A and B and GDH but in positive sample resluts further investigations such as subsequent ELISA or PCR testing are needed. 
The two- and three-step algorithmic approach for CDI testing was performed. In this approach, the samples were tested for glutamate dehydrogenase (GDH), further enzyme immunoassay for toxins A/B (immunochromatography) or enzyme immunoassay for toxins A/B (ELISA test) and real-time PCR were performed.

By two- step algorithm $-87,6 \%$ of samples could be evaluated as positive (511 biologic samples) or negative (1144 biologic samples) and could be reported within 4 hours (turnaround time).

$12,4 \%$ (233) samples were evaluated positive for glutamate dehydrogenase (GDH) test and for toxins A/B (EIA) negative. In discordant cases, in suspition of CDI with positive symptoms, the three-step algorithm was applied (i.e. real-time PCR) with exclusion of toxigenic or hypervirulent strains of C.difficile or confirmation of C.difficile toxigenic strains with aim to set up the treatment when needed.

Interpretation of laboratory findings should always be evaluated in relation to the clinical finding, other laboratory and radiological results of a particular patient in relation to the current epidemiological situation.

\section{REFERENCES}

1. Bartlett J, Clostridium difficile: history of its role as an enteric pathogen and the current state of knowledge about the organism. Clin Infect Dis 1994, 18(Suppl.4): 265-272.

2. Khanna S, The Epidemiology of Community-acquired Clostridium difficile infection: A populationbased study. Am J Gastroenterol. 2012 Jan; 107(1): 89-95.

3. Novak M, Mazakova I, Sadlonova V, Cervenova T, Hudeckova H, Appearance of Clostridium difficile infections in health care institutions in Slovakia and in the district of Martin. Acta Medica Martiniana, Vol.: 18 (2018), pp. 5-12.

4. Gateau C, Couturier J, Coia J, Barbut F, How to: diagnose infection caused by Clostridium difficile. Clinical Microbiology and Infection 24 (2018) 463-468.

5. Farrell RJ., LaMont JT. (2000). Pathogenesis and Clinical Manifestations of Clostridium difficile Diarrhea and Colitis. In: Aktories, K., Wikins, T.C. In: Clostridium difficile, Berlin, Heidelberg: Springer Verlag, pp.109-125. ISSN 0070-217X

6. Harvey RA, Champe PC, Fisher BD, Lippincott's Illustrated Reviews: Microbiology. Lippincott Williams \& Wilkins, 432 p. 2006. Available: http://fcpspartldentistry. com/lippincotts-illustrated-reviews-microbiology-2nd-edition-chm-download/

7. Novakova E, Stefkovicova M, Garabasova Kopilec M, Novak M, Kotlebova N, Kuijper EJ, Krutova M, The emergence of Clostridium difficile ribotypes 027 and 176 with a predominance of the Clostridium difficile ribotype 001 recognized in Slovakia following the European standardized Clostridium difficile infection surveillance of 2016. International Journal of Infectious Diseases 90 (2020) 111-115.

8. Nyc O, Krutova M, Liskova A, Matejkova J, Drabek J, Kuijper EJ, The emergence of Clostridium difficile PCR-ribotype 001 in Slovakia. Eur J Clin Microbiol Infect Dis 2015, 34(8), 1701-8.

9. Solomon K, The host immune response to Clostridium difficile infection Katie Solomon, Therapeutic Advances in Infectious Disease, (2013) 1(1) 19-35.

10. Neuschlová M, Nováková E, Kompaníková J, Application of e-learning in education of immunology in Jessenius Medical Faculty, In: Edulearn 15, 7th International Conference on Education and New Learning Technologies, Barcelona. IATED Academy, pp. 7739-7744, 2015.

11. Jarčuška P, Bátovský M, Drgoňa L, a spol. Odporúčaný postup diagnostiky a liečby kolitídy spôsobenej Clostridium difficile. Via pract 2015; 1: 1-12

12. Peng Z, Ling L, Stratton CW, Li C, Polage C R, Wu B, Tang YW, Emerging Microbes \& Infections (2018) 7:15

13. Crobach MJT., Planche T, Eckert C, Barbut F, Terveer EM, Dekkers OM, Wilcox MH, Kuijper EJ, European Society of Clinical Microbiology and Infectious Diseases: update of the diagnostic guidance document for Clostridium difficile infection. Clinical Microbiology and Infection 22 (2016) 63-81. 
14. Nomlomo E, Nana T, The impact of diagnostic methods on the diagnosis of Clostridiodes difficile infection. South African Medical Journal, Volume 110, Issue 2, pp. 135-139.15

15. Murad YM, False Negative Results in Clostridium difficile Testing. BMC Infect Dis. 2016; 16: 430.

16. Davies KA, Longshaw CM, Davis GL, et al. Underdiagnosis of Clostridium difficile across Europe: the European, Multicentre, Prospective, Biannual, Point-Prevalence Study of Clostridium difficile Infection in Hospitalised Patients With Diarrhoea (EUCLID). Lancet Infect Dis. 2014;14(12): 1208-1219.

17. Alfa MJ, Sepehri S, Combination of Culture, Antigen and Toxin Detection, and Cytotoxin Neutralization Assay for Optimal Clostridium difficile Diagnostic Testing. Canadian Journal of Infectious Diseases and Medical Microbiology, Volume 24, Issue 2, Pages 89-92, 2013

18. Robotham J, Wilcox M, Updated Guidance on the Diagnosis and Reporting of Clostridium Difficile. NHS, Advisory Committee on Antimicrobial Resistance and Healthcare Associated Infection (ARHAI), 2012. Available: https://www.gov.uk/ government/uploads/system/uploads/attachment_data/file/146808/dh_133016.pdf.pdf

19. Krutova M, Wilcox MH, Kuijper, EJ, The pitfalls of laboratory diagnostics of Clostridium difficile infection, Clinical Microbiology and Infection, Vol. 24, Issue 7, pp.682-683, 2018

20. Kompanikova J, Zumdick A, Neuschlova, M et al., Microbiologic Methods in the Diagnostics of Upper Respiratory Tract Pathogens, Clinical research and practice, Advances in Experimental Medicine and Biology, Vol. 1020, 25-31, 2017.

21. Debast MP, Bauer EJ, Kuijper SB, European Society of Clinical Microbiology and Infectious Diseases: update of the treatment guidance document for Clostridium difficile infection. In: Clinical Microbiology and Infection. Vol.20, Issue 2, 2014, pp. 1-26. Available: http://onlinelibrary.wiley.com/doi/10.1111/1469-0691.12418/full

22. Burnham C, Carroll KC, Diagnosis of Clostridium difficile Infection: an Ongoing Conundrum for Clinicians and for Clinical Laboratories. In: Clinical Microbiology Reviews, 2013, Vol.26, No.3, pp. 604-630

Received: February, 15, 2020

Accepted: February, 28, 2020 